\title{
Isolated Hepatic Tuberculosis Presenting as Cystic-Like and Tumour-Like Mass Lesions
}

\author{
Cem Kaan Parsak ${ }^{a}$ Ismail Hanta ${ }^{b}$ Ahmet Aslan ${ }^{c}$ \\ Omer Alabaz
}

Departments of a General Surgery and ${ }^{\mathrm{b} C h e s t ~ D i s e a s e s, ~ F a c u l t y ~ o f ~ M e d i c i n e, ~}$ Çukurova University, Balcali, and 'Department of General Surgery, Faculty of Medicine, Mustafa Kemal University, Antakya, Turkey

\section{Key Words}

Cystic liver lesions · Hepatic tuberculosis · Liver abscesses

\begin{abstract}
Hepatic tuberculosis is a rare manifestation of extra-pulmonary tuberculosis. Hepatic tuberculous lesions are especially mimicking tumour-like mass or cystic lesions in the liver and so can be misdiagnosed with several diseases. Histopathological examination of the specimen is essential in the diagnosis for hepatic tuberculosis. In this report, two cases with hepatic tuberculosis having cystic solid mass and abscess liver lesions are described.
\end{abstract}

\section{Introduction}

Hepatic tuberculosis is a rare manifestation of extra-pulmonary tuberculosis.

Tuberculous lesions of the liver may be unexpectedly detected during abdominal surgery. Also, these lesions can, mistakenly, be diagnosed as tumour-like mass or cystic lesions in the liver on screening investigations [1]. Histopathological confirmation is essential for the exact diagnosis of hepatic tuberculosis. In this study, we discuss two cases of hepatic tuberculosis due to diagnostic dilemma and rarity of these lesions.

\section{Case 1}

A 30-year-old woman was admitted to our hospital with a history of abdominal pain, anorexia, weight loss and fever for one month. On physical examination, except hepatomegaly, no other physical finding was detected. Liver function tests (ALT $64 \mathrm{U} / \mathrm{l}$, AST $76 \mathrm{U} / \mathrm{l}$ ) and alkaline phosphatase (ALP 310 U/l) were elevated. Chest $\mathrm{x}$-ray was normal. Ultrasonography (US) of the abdomen revealed a lobulated cystic mass with air in the right lobe of the liver. Computerized tomography (CT) of the abdomen 
showed an ill-defined cystic mass with air-fluid level in the anterior segments of right liver lobe (fig. 1). Liver abscess or infected hydatid disease was considered with these radiologic and clinical findings. Liver wedge biopsy was performed during laparotomy. Histopathological examination of the specimen demonstrated granulomas, epithelioid histiocytes and Langhans giant cells, and caseating granulomatous inflammation. Hepatic tuberculosis was diagnosed based on these pathologic findings. Unfortunately we did not perform culture of the specimen for tuberculosis, because tuberculosis was not taken in account in the differential diagnosis. The patient was treated with anti-tuberculous drugs for one year. She recovered clinically and abdominal US was normal after one year.

\section{Case 2}

A 63-year-old man was admitted to our hospital with abdominal pain and fever for one week. Except for abdominal tenderness, physical examination was normal. Only white blood cells $\left(16,400 / \mathrm{mm}^{3}\right)$ and liver function values (ALT $48 \mathrm{U} / \mathrm{l}$, AST $52 \mathrm{U} / \mathrm{l}$ ) were elevated in laboratory examination. Abdominal US revealed a tumour-like mass lesion on the right lobe of the liver. Liver wedge biopsy was performed from the lesion on the liver during laparotomy. Histopathological examination of the specimen demonstrated granulomas, epithelioid histiocytes and Langhans giant cells, and caseating granulomatous inflammation. The patient was treated with anti-tuberculous drugs for one year. He recovered clinically and abdominal US was normal after one year.

\section{Discussion}

Tuberculosis is the leading cause of death among infectious diseases [2]. Among extrapulmonary tuberculosis, hepatic tuberculosis is a rare form of tuberculosis. Reed et al. described three morphologic types of hepatic tuberculosis: (1) miliary tuberculosis of the liver associated with generalized miliary tuberculosis, (2) primary miliary tuberculosis of the liver without involvement of other organs, and (3) primary tuberculous granuloma or abscesses of the liver [3]. Our two cases had only hepatic tuberculosis without pulmonary infection, which we accept as type 3 .

The symptoms and signs of hepatic involvement are non-specific. Similar to our cases, hepatic tuberculosis usually presents with abdominal pain and hepatomegaly with or without jaundice. Liver function tests and alkaline phosphatase may be abnormal $[4,5]$.

MRI and CT findings of liver tuberculosis reveal different stages of disease, varying from granulomatous tubercles with or without caseation necrosis to fibrosis and calcification in the healing stage [6]. US findings of hepatic tuberculosis usually show hypoechoic lesion [7]. CT findings demonstrate miliary, hypodense nodular lesions or cystic lesion [8]. In our cases, one of the patients had cystic mass with air-fluid level, and the other patient had tumour-like mass lesion.

Histopathological examination of the specimens from lesions is essential for exact diagnosis. Percutaneous needle biopsy and laparoscopy can be used for the diagnosis and occasionally laparotomy may be required in some patients.

Infectious and non-infectious diseases which can cause caseating or non-caseating hepatic granulomatous such as leprosy, sarcoidosis, Hodgkin's disease, brucellosis, infectious mononucleosis, inflammatory bowel disease, drug-induced liver damage and syphilis should be considered in the differential diagnosis of hepatic tuberculosis. Chronic active hepatitis may also mimic tuberculosis of the liver [4].

Antituberculous treatment should be given at least for one year; however, many antituberculous drugs are hepatotoxic and may induce liver damage and jaundice. Therefore the response and toxicity should be assessed carefully during treatment. Our patients were 
successfully treated for one year with anti-tuberculous drugs, and no side effect was observed during the treatment period.

In conclusion, tuberculosis should be considered in the differential diagnosis of cystic and tumour-like mass lesions of the liver, especially in endemic regions.

Fig. 1. Ill-defined cystic mass with air-fluid level in the anterior segments of the right liver lobe in abdominal CT.

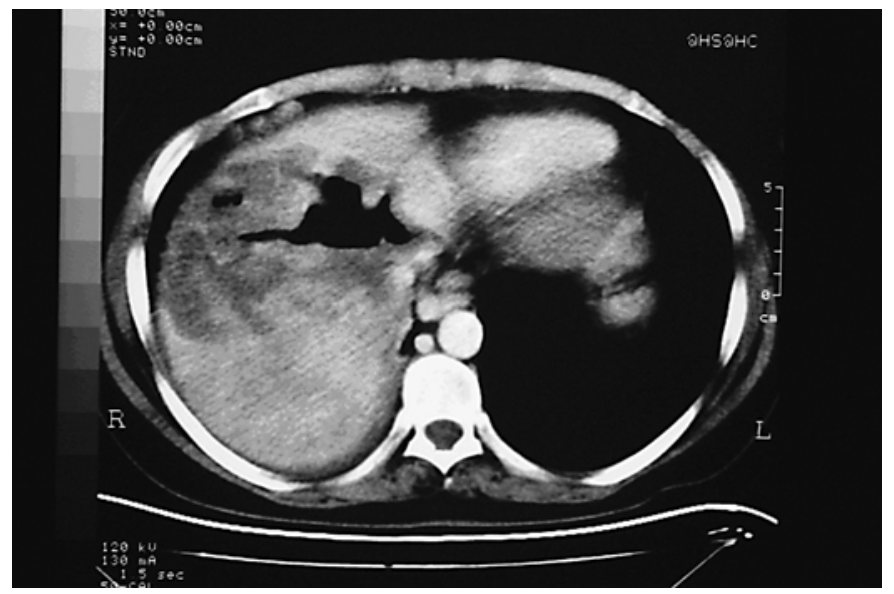




\section{References}

1 Hayashi H, Yamawaki I, Okajima K, Tomimatsu M, Ofkawa S: Tuberculous liver abscess not associated with lung involvement. Intern Med 2004;43:521-523.

-2 Corbett EL, Watt CJ, Walker N, Maher D, Williams BG, Raviglione MC, Dye C: The growing burden of tuberculosis: Global trends and interactions with the HIV epidemic. Arch Intern Med 2003;163:1009-1021.

-3 Reed DH, Nash AF, Valabhji P: Radiological diagnosis and management of a solitary tuberculous hepatic abscess. Br J Radiol 1990;63:902-904.

4 Rangabashyam N: Abdominal Tuberculosis; in Oxford Textbook of Surgery. Oxford, Oxford University Press, 1994, vol II, p 2491.

-5 Yapp SK, Kok KY: Isolated hepatic tuberculosis: report of five cases and review of the literature. J Hepatobiliary Pancreat Surg 1999;6:195-198.

-6 Kawamori Y, Matsui O, Kitagawa K, Kadoya M, Takashima T, Yamahana T: Macronodular tuberculoma of the liver: CT and MR findings. Am J Roentgenol 1992;158:311-313.

-7 Jain R, Sawhney S, Gupta RG, Acharya S: Sonographic appearances and percutaneous management of primary tuberculous liver abscess. J Clin Ultrasound 1998;27:159-163.

8 Yu RS, Zhang SZ, Wu JJ, Li RF: Imaging diagnosis of 12 patients with hepatic tuberculosis. World J Gastroenterol 2004;10:1639-1642. 